\title{
Spontaneous Small Bowel Perforations in Patients Receiving Radiotherapy or Chemotherapy for Advanced Head and Neck Cancer
}

\author{
${ }^{1}$ Muttagi Sidramesh, ${ }^{2}$ Pankaj Chaturvedi \\ ${ }^{1}$ Fellow, Department of Head and Neck Surgical Oncology, Tata Memorial Hospital, Mumbai, Maharashtra, India \\ ${ }^{2}$ Assistant Professor, Department of Surgical Oncology, Tata Memorial Hospital, Mumbai, Maharashtra, India
}

Correspondence: Pankaj Chaturvedi, Assistant Professor, Tata Memorial Hospital, EB Road, Parel, Mumbai, Maharashtra India, Phone: 91-22-24147000, Fax: 91-22-24146937, e-mail: chaturvedi.pankaj@gmail.com

\section{ABSTRACT}

Gastroduodenal perforations are known to occur due to various causes. However, such problems involving small bowel and manifesting spontaneously, especially in a head and neck cancer patient, undergoing chemoradiation has never been described in the literature. We report three cases of head and neck cancer that were undergoing treatment (one case curative and two cases palliative), during which small bowel perforation manifested requiring urgent surgical intervention. We seek to highlight this problem, so as to ascertain the possible mechanisms of its causation, identify a group of patients at risk, evolve possible preventive strategies and facilitate earlier diagnosis and treatment.

Keywords: Spontaneous, Ileal perforation, Oral cancer, Chemoradiation.

\section{INTRODUCTION}

Little is known about small bowel perforation in cancer patients receiving radical treatment. Patients with cancer have compromised nutritional, metabolic and immune conditions. Though gastroduodenal perforations are known, ${ }^{1}$ spontaneous small bowel perforation in otherwise healthy head and neck cancer patients undergoing treatment has not been reported. Review of the literature reveals a number of etiological factors responsible for small bowel perforations. ${ }^{2}$ We seek to highlight this problem, so as to ascertain the possible mechanisms of its causation, identify a group of patients at risk, evolve possible preventive strategies and facilitate earlier diagnosis and treatment.

\section{CASE REPORT}

Literature is very sparse regarding gastrointestinal (GI) perforations in non-GI cancer patients. Gastroduodenal perforations, resulting from prolonged NSAID intake or stress or other treatment related conditions, are previously reported in such patients. ${ }^{1,2}$ Literature review revealed that spontaneous small bowel perforation, especially distal ileal in non- Gl cancer patients undergoing treatment is extremely uncommon. ${ }^{3}$ We wish to know if anybody else has witnessed this problem so as to ascertain the etiology and evolve possible preventive strategy. First case was a 46-year-old male undergoing concurrent chemotherapy (carboplatin and 5-flurouracil) and radiotherapy (RT) for advanced carcinoma of the oral cavity. Patient developed pain in abdomen and distention after fifteenth fraction of RT. Patient did not have fever, bowel alteration and past history suggestive of gastroduodenal ulceration. Plain skiagram suggested perforation. Celiotomy revealed eight perforations at mesentric border in the distal ileum extending up to 30 $\mathrm{cm}$ proximal to ileocecal junction (Fig. 1). The whole segment was unhealthy and there were several areas of impending perforation. Ileocecal resection with ileocolic anastomosis covering ileostomy with peritoneal lavage with placement of four tube drains was done. Two other patients (60-year female and 50-year male) were undergoing

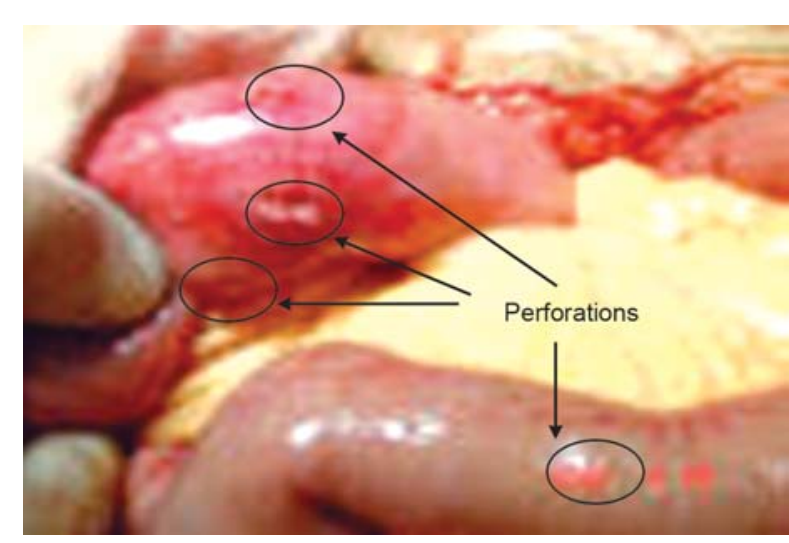

Fig. 1: Intraoperative photograph showing multiple small bowel perforations 
palliative radiotherapy for advanced carcinoma of the oral cavity. Approximately, two weeks following the radiotherapy both patients complained of pain and distention in abdomen. Plain skiagram suggested perforation. Celiotomy revealed multiple distal ileal perforation, which was resected, and ileocolic anastomosis with proximal ileostomy was done. Radiotherapy was reinstituted in all the patients as soon as possible.

All of our patients were taking NSAID frequently for their pain and two were taking steroid for severe RT reaction. They also gave history of dyspepsia and heartburn. Strikingly, Celiotomy in all cases was done with a provisional diagnosis of gastroduodenal perforation but it actually turned out to be a distal ileal perforation. All three resected bowels were sent for pathological and microbiological examinations. Same day blood culture and widal test was ordered for all patients. Widal test and blood culture ruled out typhoid etiology. Typhoid and regional enteritis are the common causes of such perforation that were ruled out in all of our cases. Microbiological examination did not show growth of Salmonella, Yersinia, enteroinvasive E. coli, Aeromonas, Mycobacteria, etc. which are known pathogens causing infective enteritis. The only positive finding in microscopy was ischemic changes in distal small bowel.

Spontaneous free perforations have been reported by Orringer et al at Lahey clinic. ${ }^{4}$ They picked up 19 such cases (in 23 years) of which 15 had history of recent use of steroid, chemotherapy or radiotherapy. Jejunal perforation in a patient receiving chemotherapy and steroid for lymphoma has also been reported. ${ }^{2}$ Two of our patient were also receiving steroid ( $8 \mathrm{mg}$ IV bid) but to our knowledge steroid is not a known causative agent for distal small bowel ulceration/perforation. Small bowel perforation due to leukemic cell infiltration ${ }^{4}$ or due to metastatic disease, e.g. breast cancer, ${ }^{5}$ lung cancer ${ }^{6}$ have also been documented. Our patients did not show evidence of metastasis to the small bowel. There is a report of small bowel perforation due to ischemia caused by systemic cholesterol atheromatous embolism. ${ }^{7,8}$ Microscopic examination of all of our specimens did not reveal any such features. Light microscopy did not show gross evidence of vasculopathy $y^{9,10}$ that could have been yet another possible cause of ischemia. All the patients were taking NSAID for their pain relief (two were taking ibuprofen and paracetamol, one was taking diclofenac) and recent evidences show that it can cause small bowel enteropathy. ${ }^{11}$ All these patients had advanced head and neck cancers, undergoing treatment when they developed perforation with strikingly similar clinicopathological picture. All the patients had surgical treatment and could be saved. There is a need to identify the causative agent which will determine the preventive strategy.

\section{REFERENCES}

1. Chao TC, Wang CS, Chen MF. Gastroduodenal perforation in cancer patients. Hepatogastroenterology Sep-Oct 1999; 46(29):2878-81.

2. Wada M, Onda M, Tokunaga A, et al. Spontaneous gastrointestinal perforation in patients with lymphoma receiving chemotherapy and steroids: Report of three cases. Nippon Ika Daigaku Zasshi Feb 1999;66(1):37-40.

3. Orringer RD, Coller JA, Veidenheimer MC. Spontaneous free perforation of the small intestine. Dis Colon Rectum May 1983;26(5):323-26.

4. Bohgaki T, Notoya A, Mukai M, Kohno M. Perforation of small intestine during hematologic recovery in an elderly man after induction therapy for acute lymphoblastic leukemia L3. Rinsho Ketsueki Feb 2000;41(2):146-51.

5. Cornu-Labat G, Ghani A, Smith DJ, McDonald AD, Kasirajan K. Small-bowel perforation secondary to metastatic carcinoma of the breast. Am Surg Apr 1998;64(4):312.

6. Yokota T, Yamada Y, Sakata N, et al. Emergency abdominal surgery for small bowel perforation secondary to metastatic lung cancer. Tohoku J Exp Med Jul 1999;188(3):265-70.

7. Fujiyama A, Mori Y, Yamamoto S, et al. Multiple spontaneous small bowel perforations due to systemic cholesterol atheromatous embolism. Intern Med Jul 1999;38(7):580-84.

8. Turnbull RG, Hayashi AH, McLean DR. Multiple spontaneous intestinal perforations from atheroembolism after thrombolytic therapy: A case report. Can J Surg Aug 1994;37(4):325-28.

9. Usuda H, Emura I, Naito M. Crystalglobulin-induced vasculopathy accompanying ischemic intestinal lesions of a patient with myeloma. Pathol Int Feb 1996;46(2):165-70.

10. Verma S, Giles TE, Tsai HH. Vasculitic small bowel perforation masquerading as spontaneous bacterial peritonitis in a patient with decompensated liver disease. Eur J Gastroenterol Hepatol Apr 1999;11(4):459-61.

11. Morris AJ. Nonsteroidal anti-inflammatory drug enteropathy. Gastrointest Endosc Clin N Am Jan 1999;9 (1):125-33. 